RECONSTRUCTION OF THE ORIENTATION DISTRIBUTION FUNCTION IN SINGLE AND MULTIPLE SHELL Q-BALL IMAGING WITHIN CONSTANT SOLID ANGLE

By

\author{
Iman Aganj \\ Christophe Lenglet \\ Guillermo Sapiro \\ Essa Yacoub \\ Kamil Ugurbil
}

and

Noam Harel

IMA Preprint Series \# 2267

(July 2009)

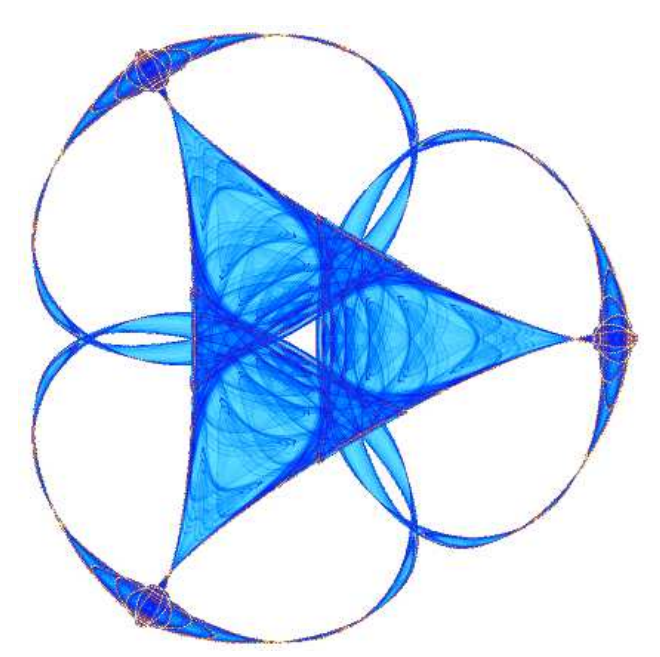

INSTITUTE FOR MATHEMATICS AND ITS APPLICATIONS

UNIVERSITY OF MINNESOTA 400 Lind Hall 207 Church Street S.E.

Minneapolis, Minnesota 55455-0436

Phone: 612-624-6066 Fax: 612-626-7370

URL: http://www.ima.umn.edu 


\section{Reconstruction of the Orientation Distribution Function in Single and Multiple Shell Q-Ball Imaging within Constant Solid Angle}

Iman Aganj, ${ }^{1}$ Christophe Lenglet, ${ }^{1,2}$ Guillermo Sapiro, ${ }^{1}$ Essa Yacoub, ${ }^{2}$ Kamil Ugurbil, ${ }^{2}$ and Noam Harel $^{2}$

${ }^{1}$ Department of Electrical and Computer Engineering, University of Minnesota, Minneapolis, MN, USA

${ }^{2}$ Center for Magnetic Resonance Research, University of Minnesota, Minneapolis, MN, USA

Author responsible for correspondence:

Iman Aganj

$309,6^{\text {th }}$ St. SE, \#212, Minneapolis, MN 55414, USA

Tel: $\quad+1(612) 202-3019$

Email: iman@umn.edu 


\begin{abstract}
Q-ball imaging (QBI) is a high angular resolution diffusion imaging (HARDI) technique which has been proven very successful in resolving multiple intravoxel fiber orientations in MR images. The standard computation of the orientation distribution function (ODF, the probability of diffusion in a given direction) from q-ball data uses linear radial projection, neglecting the change in the volume element along each direction. This results in spherical distributions that are different from the true ODFs. For instance, they are neither normalized nor as sharp as expected, and generally require post-processing, such as artificial sharpening or spherical deconvolution. In this paper, a new technique is proposed that, by considering the solid angle factor, uses the mathematically correct definition of the ODF and results in a dimensionless and normalized ODF expression. Our model is flexible enough so ODFs can either be estimated from single q-shell datasets, or exploit the greater information available from multiple q-shell acquisitions. We show that this can be achieved by using a more accurate multi-exponential model for the diffusion signal. The improved performance of the proposed method is demonstrated on artificial data and real HARDI volumes.
\end{abstract}

Key words: $\quad$ Orientation distribution function (ODF), q-ball imaging (QBI), high angular resolution diffusion imaging (HARDI), solid angle. 


\section{INTRODUCTION}

Diffusion-weighted magnetic resonance imaging (DWMRI) provides valuable information about the fiber architecture of neural tissue by measuring the diffusion of water molecules in three-dimensional (3D) space. The microscopic diffusion function may be measured by using the model-free diffusion spectrum imaging (DSI) (1), which uses the direct Fourier inversion of the diffusion signal. This technique is time intensive, as it measures the diffusion signal on a 3D (e.g., $11 \times 11 \times 11)$ Cartesian lattice. Thus, an alternative approach based on sampling only on one or multiple spherical shells in q-space has been proposed, referred to as high angular resolution diffusion imaging (HARDI) (2). The spherical shell, being a 2D manifold, includes a number of measurement points which grows quadratically with the desired angular resolution, as opposed to cubically with the spatial resolution in the entire 3D lattice of DSI.

While the 3D probability density function (PDF) of the diffusion is helpful in studying the tissue microstructure, the orientation distribution function (ODF) - the marginal probability of diffusion in a given direction - is the quantity of interest for mapping the orientation architecture of the tissue. Q-ball imaging (QBI), (3), is a widely used acquisition scheme for HARDI, from which ODFs can be reconstructed through a spherical tomographic inversion called the Funk-Radon transform. This technique's simplicity and its ability to resolve intravoxel fiber orientations have made it popular for fiber tracking and characterizing white matter architecture. A number of recently proposed methods have turned QBI into a very efficient and robust technique (4)-(6). Moreover, a few works have suggested exploiting data from multiple q-shells to benefit from the high signal-to-noise ratio (SNR) and high angular contrast-to-noise ratio (CNR) of the data acquired at respectively low and high b-values, (3), (7)(9). Using multiple q-shells also allows us to employ richer models for the diffusion signal, as discussed in this paper.

However, the definition of the ODF used in the original QBI is different from the actual marginal PDF of diffusion in a constant solid angle. It is computed as a linear radial projection of the PDF, which does not take into account the quadratic growth of the volume element with respect to its distance from the origin (see the "General ODF Definition" section and Fig. 1 for more details). This inaccurate formulation generally distorts the ODF, produces non-distribution functions, and has created the need for artificial post-processing such as manual normalization and sharpening.

In this paper, we re-derive the ODF expression for QBI via Fourier analysis, this time starting from the proper definition of the ODF in constant solid angle. We show that this results in an inherently 
normalized and dimensionless expression. In addition, we illustrate through our experiments that the ODFs are naturally sharp and that multiple fiber orientations are thus better resolved. We also provide a general formulation for multiple q-shell QBI, and demonstrate the improvement achieved by considering the information from multiple q-shells and using richer multi-exponential models. Furthermore, by making use of the spherical harmonic basis, we demonstrate that the implementation of the new, mathematically correct expression is as straightforward as that of the original formula, or maybe even simpler, considering that further sharpening (post-processing) is not necessary.

This paper extends our previous conference versions for single (10) and multiple q-shells (11). In particular, we provide complete mathematical proofs, a regularization scheme, and additional validation and comparisons. ${ }^{1}$

\section{METHODS}

\section{General ODF Definition}

The PDF of the diffusion of water molecules, $P(\vec{r})$, gives the displacement probability $P(\vec{r}) d v$ of a molecule, initially placed at the origin, to be in the infinitesimal volume $d v$ located at $\vec{r}$ after a certain amount of time. We assume this function to be symmetric (i.e. $P(-\vec{r})=P(\vec{r})$ ), which is a quite common assumption in DWMRI. The PDF can be presented in Cartesian coordinates with $\vec{r}=(x, y, z)^{T}$ and $d v=d x d y d z$. However, for mapping the orientation architecture of the tissue, the representation which mostly interests us is in the standard spherical coordinates, parameterized by $(r, \theta, \phi)$, where $\vec{r}=r \hat{u}$, with $\hat{u}(\theta, \phi)=(\sin \theta \cos \phi, \sin \theta \sin \phi, \cos \theta)^{T}$ being the unit direction vector. The volume element in this case is $d \nu=r^{2} d r d \Omega$ with $d \Omega=\sin \theta d \theta d \phi$ being the infinitesimal solid angle element.

\footnotetext{
${ }^{1}$ After our conference paper was accepted and its extension to multiple shells was submitted, a parallel and independent work was published (14), where the proper definition of the ODF was considered in single q-shell QBI. However, in addition to not considering multiple shells and the richer model as done here, the authors of (14) take the integral of the diffusion signal on a circle and not on the entire plane, and that results in a different formula which is not necessarily normalized and leads to other potential inaccuracies. (See the "Q-ball Imaging ODF Reconstruction" section for further details and comparison).
} 
We denote by $O D F(\hat{u}) d \Omega$ the probability of diffusion in the direction $\hat{u}$ through the solid angle $d \Omega$, which can be computed by integrating the displacement probabilities, i.e., $P(\vec{r}) d v=P(r \hat{u}) r^{2} d r d \Omega$, for all magnitude $r$, while keeping $\hat{u}$ constant:

$$
O D F(\hat{u}) d \Omega=\int_{r=0}^{r=\infty} P(r \hat{u}) r^{2} d r d \Omega,
$$

or simply:

$$
O D F(\hat{u})=\int_{0}^{\infty} P(r \hat{u}) r^{2} d r
$$

The above definition, which is normalized and dimensionless, is the integral of the probability values in a cone of "very small" constant solid angle (Fig. 1(a)). This correct definition was used for instance by the authors of (1) in DSI, where $P(\vec{r})$ was first computed from the diffusion data via Fourier inversion and then integrated to calculate the ODF, and also in (12)-(13) for diffusion tensor imaging (DTI), where the ODF was analytically computed. However, the original expression for ODF reconstruction in HARDI, and specifically QBI (3), is different from Eq. [1], in the sense that the integral is not weighted by the important (and mathematically correct) factor $r^{2}$ (Fig. 1(b)). To the best of our knowledge, the only paper which has so far considered this factor in (single shell) QBI, is a very recent parallel work (14) (published independently after a conference version of our paper (10) had just been accepted), where the ODF is approximated from the q-shell using Eq. [1]. (See the "Q-ball Imaging ODF Reconstruction" section for details and comparison.)

Computing the ODF without the factor $r^{2}$ would be equivalent to assuming the PDF to be $P(\vec{r}) /|\vec{r}|^{2}$, as $\int_{0}^{\infty} P(r \hat{u}) d r=\int_{0}^{\infty} \frac{P(r \hat{u})}{r^{2}} r^{2} d r$. This radial projection gives an artificial weight to $P(\vec{r})$ which is, respectively, too large and too small for points close to and far from the origin, and in fact, the computed quantity would be different just as the zeroth moment of a one-dimensional function $\bar{P}(r):=P(r \hat{u})$ is different from its second moment. For instance, a consequence of not including the factor $r^{2}$ is that the computed ODF will not be necessarily normalized, and an artificial normalization factor will be required. Moreover, the ODF will not be dimensionless, since, given that $P(\vec{r})$ has the dimension of $L^{-3}$ ( $L$ being the length dimension), the dimensions of $P(\vec{r}) r^{2} d r$ and $P(\vec{r}) d r$ are respectively 1 and $L^{-2}$. 
As an example intended for comparison, we compute the ODFs with and without $r^{2}$ in the case of DTI, with the following standard Gaussian PDF:

$$
P(\vec{r})=\frac{1}{(2 \pi)^{\frac{3}{2}}|D|^{\frac{1}{2}}} e^{-\frac{1}{2} \vec{r}^{T} D^{-1} \vec{r}},
$$

where $D$ is the covariance matrix (proportional to the diffusion tensor). The computed ODFs are:

$$
\begin{gathered}
\underset{\text { with } r^{2}}{O D}(\hat{u})=\frac{1}{4 \pi \mid D^{\frac{1}{2}}\left(\hat{u}^{T} D^{-1} \hat{u}\right)^{\frac{3}{2}}} \\
\underset{\text { without } r^{r^{2}}}{O F^{2}}=\frac{1}{Z} \frac{1}{4 \pi|D|^{\frac{1}{2}}\left(\hat{u}^{T} D^{-1} \hat{u}\right)^{\frac{1}{2}}},
\end{gathered}
$$

where $Z$ is the normalization constant that subsequently needs to be computed and considered in ODF $(\hat{u})$ (see (3)). An example of this pair of ODFs is illustrated in Fig. 2. (No min-max normalization is used in any of the figures.)

Next, we derive a closed-form expression for the ODF in QBI using the correct $r^{2}$-weighted integral.

\section{Q-ball Imaging ODF Reconstruction}

Let $E(\vec{q})$ be the 3D Fourier transform of $P(\vec{r})$. We have the values of $E(\vec{q})$ measured on a q-ball, i.e., the frequencies with constant norm $|\vec{q}|=q_{0}$, as $\tilde{E}(\hat{u}):=E\left(q_{0} \hat{u}\right)=\frac{S(\hat{u})}{S_{0}}$, where $S(\hat{u})$ is the HARDI signal and $S_{0}$ is the non diffusion-weighted (or B0) image. In addition, since the diffusion signal at $\vec{q}=0$ is $S_{0}$, one can see that $E(0)=1$. Alternatively, $E(0)$ is the zero frequency of a PDF which is its integral over the entire space, yielding 1 .

Our mathematical derivation is based on the following two fundamental facts from Fourier analysis:

- The Fourier transform of $P(\vec{r})|\vec{r}|^{2}$ is $-\nabla^{2} E(\vec{q})$, where $\nabla^{2}$ is the Laplacian operator (proof presented in Appendix A). 
- For a symmetric function $f: \mathbb{R}^{3} \rightarrow \mathbb{R}$ with the 3D Fourier transform function $\hat{f}(\vec{q})$, and for the arbitrary unit vector $\hat{u}$, we have that $\int_{0}^{\infty} f(r \hat{u}) d r=\frac{1}{8 \pi^{2}} \iint_{\hat{u}^{\perp}} \hat{f}(\vec{q}) d^{2} \vec{q}$, where $\hat{u}^{\perp}$ is the plane perpendicular to $\hat{u}$ (proof presented in Appendix B).

Combining these statements with Eq. [1] leads to

$$
O D F(\hat{u})=-\frac{1}{8 \pi^{2}} \iint_{\hat{u}^{\perp}} \nabla^{2} E(\vec{q}) d^{2} \vec{q}
$$

Now, without loss of generality, we choose our coordinates such that $\hat{z}=\hat{u}$, thus making $\hat{u}^{\perp}$ the $\mathrm{q}_{\mathrm{x}}-\mathrm{q}_{\mathrm{y}}$ plane. We then use the following expansion for the Laplacian in spherical coordinates $(q, \theta, \phi)$ :

$$
\nabla^{2} E(\vec{q})=\frac{1}{q} \frac{\partial^{2}}{\partial q^{2}}(q E)+\frac{1}{q^{2}} \nabla_{b}^{2} E
$$

where $\nabla_{b}^{2}$ is the Laplace-Beltrami operator which is defined independently of the radial component $q$, as $\nabla_{b}^{2} E=\frac{1}{\sin \theta} \frac{\partial}{\partial \theta}\left(\sin \theta \frac{\partial E}{\partial \theta}\right)+\frac{1}{\sin ^{2} \theta} \frac{\partial^{2} E}{\partial \varphi^{2}}$. The surface integral on the $\mathrm{q}_{\mathrm{x}}-\mathrm{q}_{\mathrm{y}}$ plane is computed by fixing $\theta=\frac{\pi}{2}$ and using the expression $d^{2} \vec{q}=q d q d \phi$ for the surface element, which yields

$$
\begin{aligned}
\operatorname{ODF}(\hat{z}) & =-\frac{1}{8 \pi^{2}} \int_{0}^{2 \pi} \int_{0}^{\infty} \nabla^{2} E(\vec{q}) q d q d \varphi \\
& =-\frac{1}{8 \pi^{2}} \int_{0}^{2 \pi} \int_{0}^{\infty}\left(\frac{1}{q} \frac{\partial^{2}}{\partial q^{2}}(q E)+\frac{1}{q^{2}} \nabla_{b}^{2} E\right) q d q d \varphi
\end{aligned}
$$

We can see that the integral of the first term is constant and independent of $E(\vec{q})$ and its derivatives:

$$
\begin{aligned}
& \int_{0}^{\infty}\left(\frac{1}{q} \frac{\partial^{2}}{\partial q^{2}}(q E)\right) q d q=\int_{0}^{\infty} \frac{\partial^{2}}{\partial q^{2}}(q E) d q \\
&=\left[\frac{\partial}{\partial q}(q E)\right]_{0}^{\infty}=\left[E+q E_{q}\right]_{0}^{\infty} \\
&=E(\infty)-E(0)+\left[q E_{q}\right]_{q=\infty}-\left[q E_{q}\right]_{q=0} \\
&=-1 \\
& \int_{0}^{2 \pi} \int_{0}^{\infty}\left(\frac{1}{q} \frac{\partial^{2}}{\partial q^{2}}(q E)\right) q d q d \phi=-2 \pi
\end{aligned}
$$


where the subscript indicates the partial derivative. We assumed (the standard assumptions) that the diffusion signal and its radial derivative go to zero (sufficiently fast) as $q \rightarrow \infty$, and also that the derivative is bounded at the origin. Therefore we have

$$
\operatorname{ODF}(\hat{z})=\frac{1}{4 \pi}-\frac{1}{8 \pi^{2}} \int_{0}^{2 \pi} \int_{0}^{\infty} \frac{1}{q} \nabla_{b}^{2} E(\vec{q}) d q d \varphi
$$

while $\theta=\frac{\pi}{2}$ is kept constant in the integration.

To compute the integral of the second term, the values of $E(\vec{q})$ are required in the entire q-space. The above equation could be used for example in the DSI modality, where direct computation of the ODF from the diffusion signal would eliminate the need for 3D Fourier inversion. In QBI, however, the values of $E(\vec{q})$ are not available in the entire q-space. Thus, we need to approximate $E(\vec{q})$ from the values measured on the q-ball. In this work, we consider the following radial mono-exponential model:

$$
E(\vec{q}) \cong E\left(q_{0} \hat{u}\right)_{q_{0}^{2}}^{q^{2}}=\widetilde{E}(\hat{u})_{q_{0}^{2}}^{\frac{q^{2}}{q^{2}}}
$$

where $q_{0}$ is the radius of the q-ball. This type of interpolation has been previously used and discussed in (15)-(16). An advantage of this model over the original QBI model, i.e. $E(q \hat{u}) \cong \widetilde{E}(\hat{u}) \delta\left(q-q_{0}\right)$ (see (17)), is the compatibility with $E(0)=1$.

After applying the mono-exponential assumption and a few more steps of calculations (see Appendix $\mathrm{C}$ for details), the following ODF expression can be derived:

$$
O D F(\hat{z})=\frac{1}{4 \pi}+\frac{1}{16 \pi^{2}} \int_{0}^{2 \pi} \nabla_{b}^{2} \ln (-\ln \tilde{E}(\hat{u})) d \phi
$$

Finally, rewriting the expression independently of the choice of axes, the following analytical formula can be shown to hold for the ODF:

$$
\operatorname{ODF}(\hat{u})=\frac{1}{4 \pi}+\frac{1}{16 \pi^{2}} F R T\left\{\nabla_{b}^{2} \ln (-\ln \tilde{E}(\hat{u}))\right\}
$$

where FRT is the Funk-Radon transform (18), defined as

$$
F R T\{f(\hat{u})\}:=\iint_{\hat{u}^{\perp}} f(\vec{w}) \delta(|\vec{w}|-1) d^{2} \vec{w},
$$


with $\delta(\bullet)$ the Dirac delta function.

The above ODF expression is dimensionless and intrinsically normalized, since the integrals of the first and second terms over the sphere are respectively 1 and 0 . This is in contrast to the ODF formulas used in the original QBI, i.e., $\frac{1}{Z} F R T\{\tilde{E}(\hat{u})\}$, and also in (14), where an artificial normalization factor $Z$ is needed.

Additional fundamental differences can be observed in the approach presented here, compared to (14). As we demonstrated, integration of the radial part of the Laplacian on the plane always results in a constant and does not require any model for the diffusion signal. Yet, (14) uses the Bessel approximation of the Dirac delta function which yields a variable (sometimes even negative) term. As for the integral of the tangential term of the Laplacian, we use the exponential model that is in particular consistent with $E(0)=1$, in contrast to (14) that assumes the tangential term of the Laplacian to be zero outside the q-ball (Bessel approximation again), leading to an expression rather similar to Laplace-Beltrami post-processing sharpening (19). A major disadvantage of approximating the Dirac delta function with a Bessel function while considering the factor $r^{2}$ is that, unlike for $P(\vec{r})$ which is typically concentrated near the origin, the projection of $P(\vec{r})|\vec{r}|^{2}$ may have its highest values at a certain positive radius coinciding with the side lobes of the Bessel function, reducing the accuracy of the approximation.

From Eq. [3], it can be seen that the essential quantity used in computing the ODF from the raw data is $L(\tilde{E}):=\ln (-\ln \tilde{E})$, which is plotted along with the absolute value of its derivative with respect to $\tilde{E}$, in Fig. 3. The behavior of this quantity is almost linear for the values of the signal close to $e^{-1} \approx 0.368$, which makes the reconstructed ODFs corresponding to such signals similar to those obtained by the original QBI (3) with Laplace-Beltrami sharpening. Nevertheless, $L(\tilde{E})$ becomes greatly non-linear as the range of the signal values approaches 0 or 1 (for e.g. by acquiring the data at respectively higher and lower b-values), and the advantages of Eq. [3] become obvious, particularly in resolving fiber crossings (see the "Results and Discussions" section).

\section{Implementation}

Our implementation of the ODF reconstruction makes use of the spherical harmonic (SH) basis, $Y_{k}^{m}(\hat{u})$, which is common for the analysis of HARDI data. The steps taken here to numerically compute Eq. [3] 
are similar to those described in (5). Particularly, we use the real and symmetric modified SH basis introduced in (5), where SH functions are indexed by a single parameter $j=k(k+1) / 2+m+1$, with corresponding $k_{j}$ and $m_{j}$, as follows:

$$
Y_{j}=\left\{\begin{array}{cc}
\sqrt{2} \operatorname{Re}\left\{Y_{k_{j}}^{m_{j}}\right\}, & -k_{j} \leq m_{j}<0 \\
Y_{k_{j}}^{0}, & m_{j}=0 \\
\sqrt{2} \operatorname{Im}\left\{Y_{k_{j}}^{m_{j}}\right\}, & 0<m_{j} \leq k_{j}
\end{array}\right.
$$

We adopt a minimum least square scheme to compute the modified $\mathrm{SH}$ coefficients $c_{j}$ of the double logarithm of the signal, such that

$$
\ln (-\ln \tilde{E}(\hat{u})) \approx \sum_{j=1}^{R} c_{j} Y_{j}(\hat{u}),
$$

where $R=(l+1)(l+2) / 2$, with $l$ being the order of the SH basis (we chose $l=4$ throughout our experiments). Next, since the SH elements are eigenfunctions of the Laplace-Beltrami operator, we compute $\nabla_{b}^{2} \ln (-\ln \tilde{E}(\hat{u}))$ by multiplying the coefficients $c_{j}$ by their corresponding eigenvalues, $-k_{j}\left(k_{j}+1\right)$. Then, as suggested in (5), the Funk-Radon transform is computed by multiplying the coefficients by $2 \pi P_{k_{j}}(0)$, where $P_{k}(\bullet)$ is the Legendre polynomial of degree $k$, with $P_{k}(0)=(-1)^{\frac{k}{2}} \frac{1 \times 3 \times \cdots \times(k-1)}{2 \times 4 \times \cdots \times k}$ for even $k$. Finally, given that $Y_{1}(\hat{u})=\frac{1}{2 \sqrt{\pi}}$, the SH coefficients of the ODF are derived as

$$
c_{j}^{\prime}=\left\{\begin{array}{cc}
\frac{1}{2 \sqrt{\pi}} & j=1 \\
-\frac{1}{8 \pi}(-1)^{\frac{k_{j}}{2}} \frac{1 \times 3 \times \cdots \times\left(k_{j}+1\right)}{2 \times 4 \times \cdots \times\left(k_{j}-2\right)} & j>1
\end{array}\right.
$$

By taking advantage of the $\mathrm{SH}$ framework, this implementation of the proposed technique for the true ODF is as straightforward as the one introduced in (5) for the original QBI ODF formula. Additionally, neither normalization, nor sharpening is required with this technique. This work was recently extended in (20) to impose positivity constraint and spatial regularity if further desired. 


\section{Regularization}

As mentioned before, the essential quantity used in computing the ODF from the raw data is $L(\tilde{E}):=\ln (-\ln \tilde{E})$, plotted in Fig. 3. Hence, if there is a relatively constant error $\Delta \tilde{E}$ in the diffusion data, the error introduced in the computed ODF will be proportional to the derivative of $L(\widetilde{E})$ :

$$
\Delta L(\tilde{E})=\left|\frac{d L}{d \tilde{E}}\right| \Delta \tilde{E}=\frac{\Delta \tilde{E}}{|\widetilde{E} \ln \tilde{E}|}
$$

As Fig. 3 suggests, this quantity becomes unstable for values of $\widetilde{E}$ close to 0 and 1, which subsequently amplifies the error in the diffusion data. To overcome this problem, we propose using a flexible threshold on the diffusion data in order to keep their values away from the unstable regions of $\left[0, \delta_{1}\right]$ and $\left[1-\delta_{2}, 1\right]$, where the thresholds $\delta_{1}$ and $\delta_{2}$ are manually defined. To perform this operation, we use the following function $f(\tilde{E})$, plotted in Fig. 4 for $\delta_{1}=\delta_{2}=0.15$ :

$$
f(\tilde{E})=\left\{\begin{array}{cc}
\frac{\delta_{1}}{2}, & \tilde{E}<0 \\
\frac{\delta_{1}}{2}+\frac{\tilde{E}^{2}}{2 \delta_{1}}, & 0 \leq \tilde{E}<\delta_{1} \\
\tilde{E}, & \delta_{1} \leq \tilde{E}<1-\delta_{2} \\
1-\frac{\delta_{2}}{2}-\frac{(1-\tilde{E})^{2}}{2 \delta_{2}}, & 1-\delta_{2} \leq \tilde{E}<1 \\
1-\frac{\delta_{2}}{2}, & 1 \leq \tilde{E}
\end{array}\right.
$$

Conversely, the ODF is most stable to noise when $\Delta L(\tilde{E})$ is minimum, which is achieved for $\widetilde{E}=e^{-1} \approx 0.368$. This gives us a clue on how to choose an optimum b-value in data acquisition. Particularly, in the mono-exponential model, since $\tilde{E}=e^{-b . A D C}$ where the Apparent Diffusion Coefficient (ADC) is assumed independent of the b-value, the optimum b-value, $b^{*}$, is obtained as

$$
b^{*}=\frac{1}{\langle A D C\rangle},
$$

where $\langle A D C\rangle$ is the mean $\mathrm{ADC}$ in the region of interest. Note that this result holds only in the simple model which assumes both ADC and $\Delta \tilde{E}$ to be independent of the b-value. 


\section{Extension to Multiple q-Shells}

\section{Multi-Exponential Model}

We have so far employed the proposed technique to compute the ODF from a single q-shell. However, if diffusion data are available on multiple q-shells, this technique can be applied to reconstruct the ODF while exploiting the information from all the q-shells. With more available data, richer models become practical and appealing. Here we consider the following radial multi-exponential model (see (16),(21)),

$$
E(q \hat{u}) \cong \sum_{k=1}^{N} \lambda_{k}(\hat{u}) \alpha_{k}(\hat{u})^{q^{2}}
$$

with the constraints

$$
\begin{gathered}
0<\alpha_{k}(\hat{u}), \lambda_{k}(\hat{u})<1, \\
\sum_{k=1}^{N} \lambda_{k}(\hat{u})=1,
\end{gathered}
$$

where Eq. [5] comes from the fact that $E(0)=1$. Once the values of $\lambda_{k}$ and $\alpha_{k}$ are estimated (see the "Parameter Estimation" subsection), they can be used in the following more general ODF expression, which is derived in details in Appendix D:

$$
O D F(\hat{u})=\frac{1}{4 \pi}+\frac{1}{16 \pi^{2}} F R T\left\{\nabla_{b}^{2} \sum_{k=1}^{N} \lambda_{k}(\hat{u}) \ln \left(-\ln \alpha_{k}(\hat{u})\right)\right\}
$$

The implementation is quite similar to what we explained in the "Implementation" section, with Eq. [4] being the only difference, as it now writes

$$
\sum_{k=1}^{N} \lambda_{k}(\hat{u}) \ln \left(-\ln \alpha_{k}(\hat{u})\right) \approx \sum_{j=1}^{R} c_{j} Y_{j}(\hat{u})
$$

In addition, the function $f(\tilde{E})$ introduced in the "Regularization" section can be applied to $\alpha_{k}$ s, to reduce the effect of the noise. 


\section{Parameter Estimation}

In order to approximate the diffusion signal in a direction $\hat{u}$ by a weighted sum of $N$ exponentials, we need to estimate the $2 N$ parameters $\lambda_{k}(\hat{u})$ and $\alpha_{k}(\hat{u})$, for $k=1, \ldots, N$. To estimate the parameters, at least $2 N-1$ independent equations - besides Eq. [5] - are required, which can be obtained from the HARDI signals measured on $M$ q-balls, for $M \geq 2 N-1$, as follows:

$$
\begin{gathered}
\sum_{k=1}^{N} \lambda_{k}(\hat{u}) \alpha_{k}(\hat{u})^{q_{i}^{2}}=\tilde{E}_{i}(\hat{u}) \\
i=1, \ldots, M
\end{gathered}
$$

where $\widetilde{E}_{i}(\hat{u}):=E\left(q_{i} \hat{u}\right)$ and $q_{i}$ corresponds to the $i^{\text {th }}$ q-ball. Parameterizing the problem in terms of bvalues, $b_{i}=\tau q_{i}^{2}$, and choosing the physical units such that the diffusion time becomes $\tau=1$, we obtain

$$
\begin{gathered}
\sum_{k=1}^{N} \lambda_{k}(\hat{u}) \alpha_{k}(\hat{u})^{b_{i}}=\tilde{E}_{i}(\hat{u}) \\
i=1, \ldots, M
\end{gathered} .
$$

Numerical optimization approaches such as the trust region algorithm, (22), may be employed to solve this non-linear system in the most general case. Here, however, we discuss two special cases (one familiar and one new) with analytical solutions. We continue this subsection considering a fixed direction, and therefore drop the notation $(\hat{u})$.

The mono-exponential assumption $(N=1)$ requires measurement on at least $M=1$ q-ball. $M=1$ leads to $\lambda_{1}=1$ and $\alpha_{1}=\tilde{E}_{1}^{1 / b_{1}}$. As it is shown in Appendix D, $\alpha_{1}^{\gamma}$ can also be a solution with any constant $\gamma$. Therefore, choosing $\gamma=b_{1}$ results in the solution $\alpha_{1}=\widetilde{E}_{1}$, which is consistent with what we already derived (Eq. [3]). Furthermore, if measured values are provided on more than one q-shells and the monoexponential model is still desired, then the assumption in this model (ADC being independent of the bvalue) suggests that the best exponential can be fitted by computing the average ADC across all the qballs.

Another practical case of great interest arises when we consider the aforementioned richer biexponential model ( $N=2$, see for example (23)-(24)) to reconstruct the ODFs from (at least) $M=3 \mathrm{q}-$ shells. For $M=3$, the following system of equations holds for each direction: 


$$
\begin{gathered}
\lambda \alpha^{b_{1}}+(1-\lambda) \beta^{b_{1}}=\widetilde{E}_{1} \\
\lambda \alpha^{b_{2}}+(1-\lambda) \beta^{b_{2}}=\widetilde{E}_{2} \\
\lambda \alpha^{b_{3}}+(1-\lambda) \beta^{b_{3}}=\widetilde{E}_{3} \\
0<\alpha, \beta, \lambda<1
\end{gathered}
$$

In general, this set of equations can be solved numerically. Nevertheless, an analytical solution can be derived for the particular and reasonable case when the sequence $0, b_{1}, b_{2}, b_{3}$ is an arithmetic progress (the sequence $x_{i}$ is an arithmetic progress if $x_{i}-x_{i-1}$ is constant). We describe this solution here, along with some regularization that guarantees the parameters to remain within the correct range.

Without loss of generality, let us assume $\alpha \geq \beta$, and also choose the physical units such that $b_{1}=1$, $b_{2}=2$, and $b_{3}=3$. Then,

$$
\begin{gathered}
\lambda \alpha+(1-\lambda) \beta=\widetilde{E}_{1} \\
\lambda \alpha^{2}+(1-\lambda) \beta^{2}=\widetilde{E}_{2} \\
\lambda \alpha^{3}+(1-\lambda) \beta^{3}=\widetilde{E}_{3} \\
0<\alpha, \beta, \lambda<1
\end{gathered}
$$

We first define and calculate the following two quantities:

$$
\begin{aligned}
& A:=\frac{\alpha+\beta}{2}=\frac{\widetilde{E}_{3}-\widetilde{E}_{1} \widetilde{E}_{2}}{2\left(\widetilde{E}_{2}-\widetilde{E}_{1}^{2}\right)} \\
& B:=\frac{\alpha-\beta}{2}=\sqrt{\left(\frac{\widetilde{E}_{3}-\widetilde{E}_{1} \widetilde{E}_{2}}{2\left(\widetilde{E}_{2}-\widetilde{E}_{1}^{2}\right)}\right)^{2}-\frac{\widetilde{E}_{1} \widetilde{E}_{3}-\widetilde{E}_{2}^{2}}{\widetilde{E}_{2}-\widetilde{E}_{1}^{2}}}
\end{aligned}
$$

The parameters are afterward computed as follows:

$$
\begin{aligned}
\alpha & =A+B \\
\beta & =A-B \\
\lambda & =\frac{1}{2}+\frac{\widetilde{E}_{1}-A}{2 B}
\end{aligned}
$$

However, we still need to ensure that they are real and in the correct ranges. One can verify that these conditions are satisfied by enforcing the following constraints: 


$$
\begin{gathered}
0<\widetilde{E}_{3}<\widetilde{E}_{2}<\widetilde{E}_{1}<1 \\
\widetilde{E}_{1}^{2}<\widetilde{E}_{2} \\
\widetilde{E}_{2}^{2}<\widetilde{E}_{1} \widetilde{E}_{3} \\
\widetilde{E}_{3}-\widetilde{E}_{1} \widetilde{E}_{2}<\widetilde{E}_{2}-\widetilde{E}_{1}^{2}+\widetilde{E}_{1} \widetilde{E}_{3}-\widetilde{E}_{2}^{2}
\end{gathered}
$$

Thus, we can obtain the optimal values of $\alpha, \beta$, and $\lambda$, by initially projecting $\tilde{E}_{i}$ s onto the subspace defined by the above inequalities, and then computing the parameters. Note that such projection is usually necessary, because the bi-exponential model may not be fully accurate and the data may be noisy. Furthermore, using a small separating margin of $\delta=0.01 \sim 0.1$ in the inequalities makes the ODFs more stable in practice.

\section{RESULTS AND DISCUSSIONS}

\section{Results from Single q-Shell}

To validate our approach, we first show results using artificial data. We simulated fiber crossing by generating diffusion images from the sum of two exponentials, $\tilde{E}(\hat{u})=\left(e^{-\hat{u}^{T} D_{1}^{\hat{u}}}+e^{-\hat{u}^{T} D_{2} \hat{u}}\right) / 2$, where $D_{1}$ is a diagonal matrix with diagonal entries $(9,2,2)$, and $D_{2}$ is $D_{1}$ rotated about the $y$-axis by a varying angle, producing diffusion values smaller than 0.15 (corresponding to rather high b-values). The ODFs were reconstructed in the fourth order $\mathrm{SH}$ basis from 76 diffusion directions, uniformly sampled on the sphere. The results are shown in Fig. 5, for three different methods: our proposed framework, the original (standard) QBI, and the original QBI followed by Laplace-Beltrami sharpening, $\left(1-\lambda \nabla_{b}^{2}\right)$ (see (19)), with parameter $\lambda=0.15$ (chosen to produce the optimal results). As can be seen, our method resolves the crossings starting at about $45^{\circ}$, compared to about $60^{\circ}$ by the other two methods. We also verified this using the dip test (25) - a measure of multimodality in a distribution - on the reconstructed ODFs from the same synthetic diffusion signals, with Rician noise. As can be observed in Fig. 6, the two modes of the ODFs with high SNR are distinguished at a crossing angle which is about $15^{\circ}$ smaller with the proposed reconstruction method, compared to the two other techniques. As expected, this difference becomes less marked as the noise increases.

We also tested our method on three real HARDI datasets; first on the physical phantom in (26), which was constructed from excised rat spinal cords and designed to have crossing tracts (90 diffusion images at 
$\mathrm{b}=1300 \mathrm{~s} / \mathrm{mm}^{2}$ ), and then on human brain data (27) (200 diffusion images at $\mathrm{b}=3000 \mathrm{~s} / \mathrm{mm}^{2}$ ). (For the third real dataset, set the "Results from Multiple q-Shells" section.) The ODFs were reconstructed with the fourth order SH basis using three approaches: our proposed method, the original (standard) QBI, and the original QBI followed by Laplace-Beltrami sharpening with parameters 0.5 for the rat data and 0.8 for the brain data. Results are superimposed on the generalized fractional anisotropy (GFA) map and presented in Fig. 7. (Note that the ODFs are shown as they are; no min-max normalization is used in any of the figures.) Our method (left) produces sharper and more accurate ODFs than the original QBI (middle). In addition, although sharpening (right) enhances the original QBI ODFs considerably in anisotropic tissue, it causes significant instability in isotropic regions (e.g. the background of the rat phantom and the CSF in the human brain data), in contrast to our technique which preserves isotropy fairly well. For the human brain dataset, we focus on the region of the centrum semiovale, where three major fiber bundles intersect: the internal capsule (IC)/corona radiata (CR), the radiations of the corpus callosum (CC), and the superior longitudinal fasciculus (SLF).

\section{Results from Multiple q-Shells}

To demonstrate the advantages of exploiting multiple q-shells in QBI, we first show experimental results on a synthetic example which consists of large diffusion values in two orthogonal directions. We synthesized diffusion images by sampling the sum of two exponentials, $E(\vec{q})=\left(|\sin \phi|^{q^{2} / 2}+|\cos \phi|^{q^{2} / 2}\right) / 2$, on seven q-shells $\left(b=q^{2}=1,2, \ldots, 7\right)$ and in 76 directions, uniformly distributed on the sphere. Figure 8 illustrates the ODFs reconstructed from single q-shells for different b-values, and from three q-shells with both mono-exponential and bi-exponential models. As can be observed, for the data acquired at low bvalues $(b=1,2,3)$, the proposed bi-exponential model using three q-shells is the only method correctly resolving the horizontal and vertical ODF peaks, corresponding to the strong ADC values in those directions $\left(\phi=0^{\circ}, 90^{\circ}, 180^{\circ}, 270^{\circ}\right)$. It should be noted, however, that the drawback of such a more general model is its lesser robustness to noise, as low order models are often more robust (e.g., computing the average of a signal is more robust than estimating the actual signal). Dark red represents negative values. These values do not appear often in general, nonetheless, a possible formal approach to handle them can be found at (20).

We also tested our method on the real HARDI dataset initially introduced in (28). An anesthetized Macaca mulatta monkey was scanned using a 7T MR scanner (Siemens) equipped with a head gradient 
coil (80mT/m G-maximum, 200mT/m/ms) with a diffusion weighted spin-echo EPI sequence. Diffusion images were acquired (twice during the same session, and then averaged) over 100 directions uniformly distributed on the sphere. We used three b-values of 1000, 2000, and $3000 \mathrm{~s} / \mathrm{mm}^{2}$, TR/TE of 4600/65 ms, and a voxel size of $1 \times 1 \times 1 \mathrm{~mm}^{3}$. The proposed method was used to reconstruct the ODFs from the three qshells using both bi-exponential and mono-exponential methods, and also from the single q-shells individually. Figure 9 depicts the results on a coronal slice through the centrum semiovale area, superimposed on the fractional anisotropy (FA) map. (For comparison, one of the sub-figures shows results by the original QBI.) Note how using the bi-exponential method allows for more clear recovery of certain fiber bundles, such as callosal radiations and corticospinal tract, and better resolution of crossing areas (see outlined regions in Fig. 9).

\section{ACKNOWLEDGMENTS}

This work was partly supported by NIH (P41 RR008079, P30 NS057091, R01 EB007813, R01 EB008645, CON000000004051-3014), NSF, the Keck Foundation, ONR, NGA, ARO, and DARPA. We would like to thank Jennifer Campbell of the McConnell Brain Imaging Centre, McGill University, and the authors of (27) for providing us with the real HARDI data. The dip test was performed using a public Matlab code written by F. Mechler. 


\section{APPENDIX A}

\section{Fourier Transform of $P(\vec{r})|\vec{r}|^{2}$}

From the Fourier analysis, we know that if $E(\vec{q})$ is the Fourier transform function of $P(\vec{r})$, then:

$$
\begin{gathered}
F\{x P(\vec{r})\}=i \frac{\partial}{\partial q_{x}} E(\vec{q}) \\
F\left\{x^{2} P(\vec{r})\right\}=-\frac{\partial^{2}}{\partial q_{x}^{2}} E(\vec{q})
\end{gathered}
$$

where $F\{\bullet\}$ is the Fourier transform operator. By writing the second equation for $y$ and $z$ and summing them, we will get:

$$
F\left\{r^{2} P(\vec{r})\right\}=-\nabla^{2} E(\vec{q})
$$

\section{APPENDIX B}

\section{Computing the Radial Projection of a Symmetric Function in the Fourier Domain}

Let $f: \mathbb{R}^{3} \rightarrow \mathbb{R}$ be a symmetric function with the 3D Fourier transform function $\hat{f}(\vec{q})$, and $\hat{u}$ be an arbitrary unit vector. We will show that $\int_{0}^{\infty} f(r \hat{u}) d r=\frac{1}{8 \pi^{2}} \iint_{\hat{u}^{\perp}} \hat{f}(\vec{q}) d^{2} \vec{q}$, where $\hat{u}^{\perp}$ is the plane perpendicular to $\hat{u}$.

Without loss of generality, we choose our coordinates such that $\hat{z}=\hat{u}$, thus making $\hat{u}^{\perp}$ the $\mathrm{q}_{\mathrm{x}}-\mathrm{q}_{\mathrm{y}}$ plane. We first rewrite the expression as a volume integral over the entire space, with the help of Dirac delta functions:

$$
\int_{0}^{\infty} f(r \hat{z}) d r=\int_{0}^{\infty} f(0,0, z) d z=\frac{1}{2} \iiint_{R^{3}} f(x, y, z) \delta(x) \delta(y) d x d y d z
$$


where the factor $\frac{1}{2}$ is required because we need the integral only on the positive half of the $z$-axis, and the function is symmetric. Let us define $g(x, y, z):=\delta(x) \delta(y)$. For the two functions $f, g: \mathbb{R}^{3} \rightarrow \mathbb{R}$ with Fourier transform functions $\hat{f}(\vec{q})$ and $\hat{g}(\vec{q})$, Parseval's theorem states that

$$
\iiint_{R^{3}} f(x, y, z) g^{*}(x, y, z) d x d y d z=\frac{1}{(2 \pi)^{3}} \iiint_{R^{3}} \hat{f}\left(q_{x}, q_{y}, q_{z}\right) \hat{g}^{*}\left(q_{x}, q_{y}, q_{z}\right) d q_{x} d q_{y} d q_{z}
$$

Computing $\hat{g}\left(q_{x}, q_{y}, q_{z}\right)=2 \pi \delta\left(q_{z}\right)$ and replacing it in the above equations, leads to

$$
\begin{aligned}
\int_{0}^{\infty} f(r \hat{z}) d r & =\frac{1}{2} \iiint_{R^{3}} f(x, y, z) g(x, y, z) d x d y d z \\
& =\frac{1}{2(2 \pi)^{3}} \iiint_{R^{3}} \hat{f}\left(q_{x}, q_{y}, q_{z}\right) 2 \pi \delta\left(q_{z}\right) d q_{x} d q_{y} d q_{z} \\
& =\frac{1}{8 \pi^{2}} \int_{-\infty}^{\infty} \int_{-\infty}^{\infty} \hat{f}\left(q_{x}, q_{y}, 0\right) d q_{x} d q_{y}
\end{aligned}
$$

As can be seen, the integral is taken on the $\mathrm{q}_{\mathrm{x}}-\mathrm{q}_{\mathrm{y}}$ plane, which is $\hat{u}^{\perp}$. This completes the proof.

\section{APPENDIX C}

\section{Incorporating the Mono-Exponential Model in the ODF Formula}

We will show here that by assuming the mono-exponential model, $E(\vec{q})=\widetilde{E}(\hat{u})_{q_{0}}^{q^{2}}$, we have:

$$
\int_{0}^{2 \pi} \int_{0}^{\infty} \frac{1}{q} \nabla_{b}^{2} E(\vec{q}) d q d \phi=-\frac{1}{2} \int_{0}^{2 \pi} \nabla_{b}^{2} \ln (-\ln \tilde{E}(\hat{u})) d \phi
$$

while $\theta=\frac{\pi}{2}$ is kept constant in the integration. We begin by proving a lemma:

Lemma: For a continuous and differentiable function $f(\theta, \varphi): S^{2} \rightarrow \mathbb{R}$ with $S^{2}$ being the unit sphere, we have:

$$
\int_{0}^{2 \pi} \nabla_{b}^{2} f\left(\frac{\pi}{2}, \phi\right) d \phi=\int_{0}^{2 \pi} f_{\theta \theta}\left(\frac{\pi}{2}, \phi\right) d \phi
$$

where the subscript indicates the partial derivative.

Proof: We use the following expansion for Laplace-Beltrami operator: 


$$
\begin{aligned}
\nabla_{b}^{2} f & =\frac{1}{\sin \theta} \frac{\partial}{\partial \theta}\left(\sin \theta \frac{\partial f}{\partial \theta}\right)+\frac{1}{\sin ^{2} \theta} \frac{\partial^{2} f}{\partial \phi^{2}} \\
& =\cot \theta \cdot f_{\theta}+f_{\theta \theta}+\frac{1}{\sin ^{2} \theta} f_{\phi \phi} \\
\left.\nabla_{b}^{2} f\right|_{\theta=\frac{\pi}{2}} & =f_{\theta \theta}+f_{\phi \phi}
\end{aligned}
$$

The integral of the second term is zero, because of the periodicity of $f_{\phi}$ :

$$
\int_{0}^{2 \pi} f_{\phi \phi}\left(\frac{\pi}{2}, \phi\right) d \phi=\left.f_{\phi}\left(\frac{\pi}{2}, \phi\right)\right|_{0} ^{2 \pi}=0
$$

Therefore the only remaining term in the integral is $f_{\theta \theta}$, which completes the proof of the lemma.

We now change the orders of the integrals twice, while using the lemma in between:

$$
\begin{aligned}
\int_{0}^{2 \pi} \int_{0}^{\infty} \frac{1}{q} \nabla_{b}^{2} E(\vec{q}) d q d \phi & =\int_{0}^{\infty} \frac{d q}{q} \int_{0}^{2 \pi} \nabla_{b}^{2} E(\vec{q}) d \phi \\
& =\int_{0}^{\infty} \frac{d q}{q} \int_{0}^{2 \pi} E_{\theta \theta}(\vec{q}) d \phi \\
& =\int_{0}^{\infty} \frac{d q}{q} \frac{\partial}{\partial \theta} \int_{0}^{2 \pi} E_{\theta}(\vec{q}) d \phi \\
& =\int_{0}^{2 \pi} \frac{\partial}{\partial \theta} \int_{0}^{\infty} \frac{1}{q} E_{\theta}(\vec{q}) d q d \phi
\end{aligned}
$$

Next, we compute the radial integral:

$$
\begin{aligned}
\int_{0}^{\infty} \frac{1}{q} E_{\theta}(\vec{q}) d q & =\int_{0}^{\infty} \frac{1}{q}\left(\tilde{E}(\hat{u})_{q_{0}{ }^{2}}^{q^{2}}\right)_{\theta} d q \\
& =\int_{0}^{\infty} \frac{1}{q}\left(e^{\frac{q^{2}}{q_{0}^{2}} \ln \tilde{E}(\hat{u})}\right)_{\theta} d q \\
& =(\ln \tilde{E}(\hat{u}))_{\theta} \int_{0}^{\infty} \frac{q}{q_{0}^{2}} e^{\frac{q^{2}}{q_{0}^{2}} \ln \tilde{E}(\hat{u})} d q \\
& =\left.\frac{(\ln \tilde{E}(\hat{u}))_{\theta}}{2 \ln \widetilde{E}(\hat{u})} e^{\frac{q^{2}}{q_{0}^{2}} \ln \tilde{E}(\hat{u})}\right|_{0} ^{\infty}
\end{aligned}
$$

We know that $0<\tilde{E}(\hat{u})<1 \rightarrow \ln \tilde{E}(\hat{u})<0$, so the above expression vanishes as $q \rightarrow \infty$. Also, since for a negative function $f(\theta)$ we have $\frac{f_{\theta}(\theta)}{f(\theta)}=[\ln \mid f(\theta)]_{\theta}=[\ln (-f(\theta))]_{\theta}$, the above integral simplifies as: 


$$
\int_{0}^{\infty} \frac{1}{q} E_{\theta}(\vec{q}) d q=-\frac{1}{2}[\ln (-\ln \tilde{E}(\hat{u}))]_{\theta}
$$

Substituting in Eq. [6]:

$$
\begin{aligned}
\int_{0}^{2 \pi} \int_{0}^{\infty} \frac{1}{q} \nabla_{b}^{2} E(\vec{q}) d q d \phi & =-\frac{1}{2} \int_{0}^{2 \pi}[\ln (-\ln \tilde{E}(\hat{u}))]_{\theta \theta} d \phi \\
& =-\frac{1}{2} \int_{0}^{2 \pi} \nabla_{b}^{2} \ln (-\ln \tilde{E}(\hat{u})) d \phi
\end{aligned}
$$

We completed the proof by reusing the lemma in the last step.

\section{APPENDIX D}

\section{Incorporating the Multi-Exponential Model in the ODF Formula}

By assuming the multi-exponential model, $E(q \hat{u}) \cong \sum_{k=1}^{N} \lambda_{k}(\hat{u}) \alpha_{k}(\hat{u})^{q^{2}}$, we will show that:

$$
\int_{0}^{2 \pi} \int_{0}^{\infty} \frac{1}{q} \nabla_{b}^{2} E(\vec{q}) d q d \phi=-\frac{1}{2} \int_{0}^{2 \pi} \nabla_{b}^{2} \sum_{k=1}^{N} \lambda_{k}(\hat{u}) \ln \left(-\ln \alpha_{k}(\hat{u})\right) d \phi
$$

while $\theta=\frac{\pi}{2}$ is kept constant in the integration. The ODF will then be derived by replacing the above expression in Eq. [2].

The proof is an extension of Appendix C. We first introduce the new non-negative variable $s_{k}(\hat{u}):=-\ln \alpha_{k}(\hat{u})$ which yields $E(q \hat{u})=\sum_{k=1}^{N} \lambda_{k}(\hat{u}) e^{-s_{k}(\hat{u}) q^{2}}$. For simplicity, here we drop the notation $(\hat{u})$. We then continue from Eq. [6] and compute the radial integral

$$
\begin{aligned}
\int_{0}^{\infty} \frac{1}{q} E_{\theta} d q & =\int_{0}^{\infty} \frac{1}{q}\left(\sum_{k=1}^{N} \lambda_{k} e^{-s_{k} q^{2}}\right)_{\theta} d q \\
& =-\int_{0}^{\infty} \sum_{k=1}^{N} \lambda_{k} s_{k \theta} e^{-s_{k} q^{2}} q d q+\int_{0}^{\infty} \frac{1}{q} \sum_{k=1}^{N} \lambda_{k \theta} e^{-s_{k} q^{2}} d q .
\end{aligned}
$$

The first integral is computed the same way as in Appendix C: 


$$
\begin{aligned}
-\int_{0}^{\infty} \sum_{k=1}^{N} \lambda_{k} s_{k \theta} e^{-s_{k} q^{2}} q d q & =-\sum_{k=1}^{N} \lambda_{k} s_{k \theta} \int_{0}^{\infty} q e^{-s_{k} q^{2}} d q \\
& =\left.\sum_{k=1}^{N} \lambda_{k} \frac{s_{k \theta}}{2 s_{k}} e^{-s_{k} q^{2}}\right|_{0} ^{\infty} \\
& =-\frac{1}{2} \sum_{k=1}^{N} \lambda_{k}\left(\ln s_{k}\right)_{\theta}
\end{aligned}
$$

Regarding the second integral of Eq. [7], let us define $I(\vec{s}):=\int_{0}^{\infty} \frac{1}{q} \sum_{k=1}^{N} \lambda_{k \theta} e^{-s_{k} q^{2}} d q$, with $\vec{s}$ the vector of $s_{k} \mathrm{~S}$, and derive it with respect to $s_{k}$ :

$$
\begin{aligned}
\frac{\partial}{\partial s_{k}} I(\vec{s}) & =-\int_{0}^{\infty} q \lambda_{k \theta} e^{-s_{k} q^{2}} d q \\
& =\left.\frac{\lambda_{k \theta}}{2 s_{k}} e^{-s_{k} q^{2}}\right|_{0} ^{\infty} \\
& =-\frac{\lambda_{k \theta}}{2 s_{k}} \\
& =\frac{\partial}{\partial s_{k}}\left(-\frac{\lambda_{k \theta}}{2} \ln s_{k}\right)
\end{aligned}
$$

Then, we can see that:

$$
I(\vec{s})=-\frac{1}{2} \sum_{k=1}^{N} \lambda_{k \theta} \ln s_{k}+C
$$

where $C$ is independent of $\vec{s}$. By evaluating the function for $\vec{s}=1$ (vector of all 1s), we obtain $C=I(1)$, which we then compute using the original definition of $I(\vec{s})$ :

$$
\begin{aligned}
I(1) & =\int_{0}^{\infty} \frac{1}{q} \sum_{k=1}^{N} \lambda_{k \theta} e^{-q^{2}} d q \\
& =\int_{0}^{\infty} \frac{1}{q}\left(\sum_{k=1}^{N} \lambda_{k}\right)_{\theta} e^{-q^{2}} d q \\
& =\int_{0}^{\infty} \frac{1}{q}(1)_{\theta} e^{-q^{2}} d q \\
& =0
\end{aligned}
$$

Therefore,

$$
I(\vec{s})=-\frac{1}{2} \sum_{k=1}^{N} \lambda_{k \theta} \ln s_{k}
$$


We now insert the values of the two integrals in Eq. [7]:

$$
\begin{aligned}
\int_{0}^{\infty} \frac{1}{q} E_{\theta} d q & =-\frac{1}{2} \sum_{k=1}^{N} \lambda_{k}\left(\ln s_{k}\right)_{\theta}-\frac{1}{2} \sum_{k=1}^{N} \lambda_{k \theta} \ln s_{k} \\
& =-\frac{1}{2}\left(\sum_{k=1}^{N} \lambda_{k} \ln s_{k}\right)_{\theta} \\
& =-\frac{1}{2}\left(\sum_{k=1}^{N} \lambda_{k} \ln \left(-\ln \alpha_{k}\right)\right)_{\theta}
\end{aligned}
$$

Finally, substituting in Eq. [6]:

$$
\begin{aligned}
\int_{0}^{2 \pi} \int_{0}^{\infty} \frac{1}{q} \nabla_{b}^{2} E(\vec{q}) d q d \phi & =-\frac{1}{2} \int_{0}^{2 \pi}\left[\sum_{k=1}^{N} \lambda_{k}(\hat{u}) \ln \left(-\ln \alpha_{k}(\hat{u})\right)\right]_{\theta \theta} d \phi \\
& =-\frac{1}{2} \int_{0}^{2 \pi} \nabla_{b}^{2} \sum_{k=1}^{N} \lambda_{k}(\hat{u}) \ln \left(-\ln \alpha_{k}(\hat{u})\right) d \phi
\end{aligned}
$$

We completed the proof by using the lemma introduced in Appendix C.

An interesting observation is that if $\alpha_{k}(\hat{u})$ is a set of estimated parameters in the multi-exponential model, then for any constant $\gamma$, the set $\alpha_{k}(\hat{u})^{\gamma}$ results in the same ODF:

$$
\begin{aligned}
\nabla_{b}^{2} \sum_{k=1}^{N} \lambda_{k}(\hat{u}) \ln \left(-\ln \alpha_{k}(\hat{u})^{\gamma}\right) & =\nabla_{b}^{2} \sum_{k=1}^{N} \lambda_{k}(\hat{u}) \ln \left(-\gamma \ln \alpha_{k}(\hat{u})\right) \\
& =\nabla_{b}^{2} \sum_{k=1}^{N} \lambda_{k}(\hat{u})\left[\ln \left(-\ln \alpha_{k}(\hat{u})\right)+\ln \gamma\right] \\
& =\nabla_{b}^{2} \sum_{k=1}^{N} \lambda_{k}(\hat{u}) \ln \left(-\ln \alpha_{k}(\hat{u})\right)+\nabla_{b}^{2}\left(\ln \gamma \sum_{k=1}^{N} \lambda_{k}(\hat{u})\right) \\
& =\nabla_{b}^{2} \sum_{k=1}^{N} \lambda_{k}(\hat{u}) \ln \left(-\ln \alpha_{k}(\hat{u})\right)+\nabla_{b}^{2} \ln \gamma \\
& =\nabla_{b}^{2} \sum_{k=1}^{N} \lambda_{k}(\hat{u}) \ln \left(-\ln \alpha_{k}(\hat{u})\right)
\end{aligned}
$$

This is expected, since the ODF is dimensionless and should not depend on the physical units of $q$. 


\section{REFERENCES}

1. Wedeen VJ, Hagmann P, Tseng WI, Reese TG, Weisskoff RM. Mapping complex tissue architecture with diffusion spectrum magnetic resonance imaging. Magnetic Resonance in Medicine. 2005;54(6):1377-1386.

2. Tuch DS, Reese TG, Wiegell MR, Makris N, Belliveau JW, Wedeen VJ. High angular resolution diffusion imaging reveals intravoxel white matter fiber heterogeneity. Magnetic Resonance in Medicine. 2002;48(4):577-582.

3. Tuch DS. Q-ball imaging. Magnetic Resonance in Medicine. 2004;52(6):1358-1372.

4. Anderson AW. Measurement of fiber orientation distributions using high angular resolution diffusion imaging. Magnetic Resonance in Medicine. 2005;54(5):1194-1206.

5. Descoteaux M, Angelino E, Fitzgibbons S, Deriche R. Regularized, fast, and robust analytical q-ball imaging. Magnetic Resonance in Medicine. 2007;58(2):497-510.

6. Hess CP, Mukherjee P, Han ET, Xu D, Vigneron DB. Q-ball reconstruction of multimodal fiber orientations using the spherical harmonic basis. Magnetic Resonance in Medicine. 2006;56(1):104117.

7. Khachaturian M, Wisco J, Tuch D. Boosting the sampling efficiency of q-ball imaging using multiple wavevector fusion. Magnetic Resonance in Medicine. 2007;57(2):289-296.

8. Wu Y, Field A, Alexander A. Computation of diffusion function measures in q-space using magnetic resonance hybrid diffusion imaging. IEEE Transactions on Medical Imaging. 2008;27(6):858-865.

9. Descoteaux M, Deriche R, Le Bihan D, Mangin J, Poupon C. Diffusion Propagator Imaging: Using Laplace's equation and multiple shell acquisitions to reconstruct the diffusion propagator. In: Proc. of $21^{\text {st }}$ Intl. Conf. on IPMI; 2009; Williamsburg.

10. Aganj I, Lenglet C, Sapiro G. ODF reconstruction in q-ball imaging with solid angle consideration. In: Proc. of $6^{\text {th }}$ IEEE ISBI; 2009; Boston.

11. Aganj I, Lenglet C, Sapiro G, Yacoub E, Ugurbil K, Harel N. Multiple q-shell ODF reconstruction in q-ball imaging. In: Proc. $12^{\text {th }}$ Intl. Conf. MICCAI; 2009; London.

12. Robinson EC, Valstar M, Hammers A, Ericsson A, Edwards AD, Rueckert D. Multivariate statistical analysis of whole brain structural networks obtained using probabilistic tractography. In: Proc. $11^{\text {th }}$ Intl. Conf. MICCAI; 2008; New York City. p. 486-493.

13. Aganj I, Lenglet C, Keriven R, Sapiro G, Harel N, Thompson PM. A Hough transform global approach to diffusion MRI tractography. In: Proc. of $17^{\text {th }}$ Annual Meeting of ISMRM; 2009; 
Honolulu.

14. Tristán-Vega A, Westin CF, Aja-Fernández S. Estimation of fiber orientation probability density functions in high angular resolution diffusion imaging. NeuroImage. 2009;47(2):638-650.

15. Stejskal EO, Tanner JE. Spin diffusion measurements: spin echoes in the presence of a timedependent field gradient. Journal of Chemical Physics. 1965;42(1):288-292.

16. Özarslan E, Shepherd TM, Vemuri BC, Blackband SJ, Mareci TH. Resolution of complex tissue microarchitecture using the diffusion orientation transform (DOT). NeuroImage. 2006;31:1086-1103.

17. Assemlal HE, Tschumperlé D, Brun L. Efficient computation of PDF-based characteristics from diffusion MR signal. In: Proc. $11^{\text {th }}$ Intl. Conf. MICCAI; 2008; New York. p. 70-78.

18. Funk P. Über eine geometrische Anwendung der Abelschen Integralgleichung. Mathematische Annalen. 1916;77:129-135.

19. Descoteaux M, Angelino E, Fitzgibbons S, Deriche R. A linear and regularized ODF estimation algorithm to recover multiple fibers in q-ball imaging. Research report 5768. INRIA; 2005.

20. Goh A, Lenglet C, Thompson P, Vidal R. Estimating orientation distribution functions with probability density constraints and spatial regularity. In: Proc. $12^{\text {th }}$ Intl. Conf. MICCAI; 2009; London.

21. Ronen I, Kim K, Garwood M, Ugurbil K, Kim D. Conventional DTI vs. slow and fast diffusion tensors in cat visual cortex. Magnetic Resonance in Medicine. 2003;49(5):785-790.

22. Branch M, Coleman T, Li Y. A subspace, interior, and conjugate gradient method for large-scale bound-constrained minimization problems. SIAM Journal on Scientific Computing. 1999;21(1):1-23.

23. Niendorf T, Dijkhuizen RM, Norris DG, Campagne ML, Nicolay K. Biexponential difision attenuation in various states of brain tissue: Implications for diffusion-weighted imaging. Magnetic Resonance in Medicine. 1996;36(6):847-857.

24. Yeh C, Cho K, Lin H, Wang J, Lin C. Reduced encoding diffusion spectrum imaging implemented with a bi-Gaussian model. IEEE Transactions on Medical Imaging. 2008;27(10):1415-1424.

25. Hartigan JA, Hartigan PM. The dip test of unimodality. The Annals of Statistics. 1985;13(1):70-84.

26. Campbell JSW, Siddiqi K, Rymar VV, Sadikot AF, Pike GB. Flow-based fiber tracking with diffusion tensor and q-ball data: Validation and comparison to principal diffusion direction techniques. NeuroImage. 2005;27(4):725-736.

27. Poupon C, Poupon F, Allirol L, Mangin JF. A database dedicated to anatomo-functional study of 
human brain connectivity. In: Proc. of the $12^{\text {th }}$ Annual Meeting of OHBM; 2006.

28. Lenglet C, Yacoub E, Ghose G, Adriany G, Krüger G, Sapiro G, Ugurbil K, Harel N. High resolution diffusion MRI on in-vivo monkey brains at 7T. In: Proc. $14^{\text {th }}$ Annual Meeting of OHBM; 2009; San Francisco. 


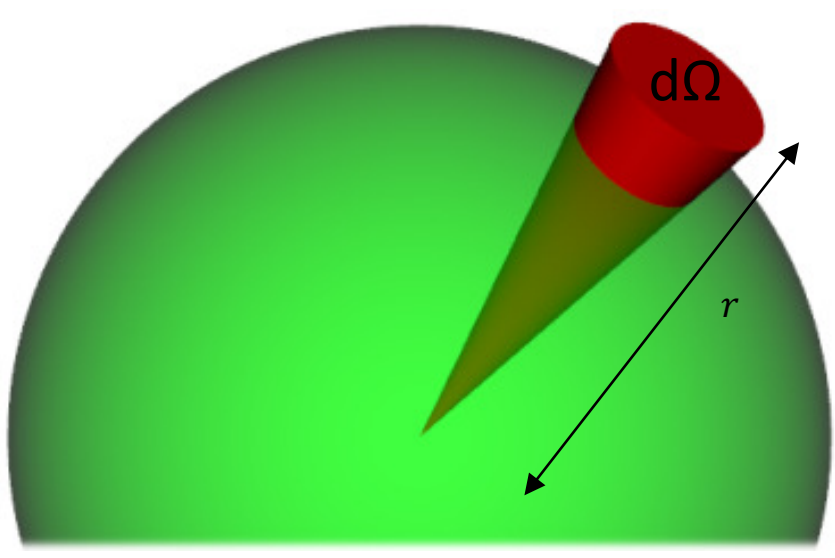

(a)

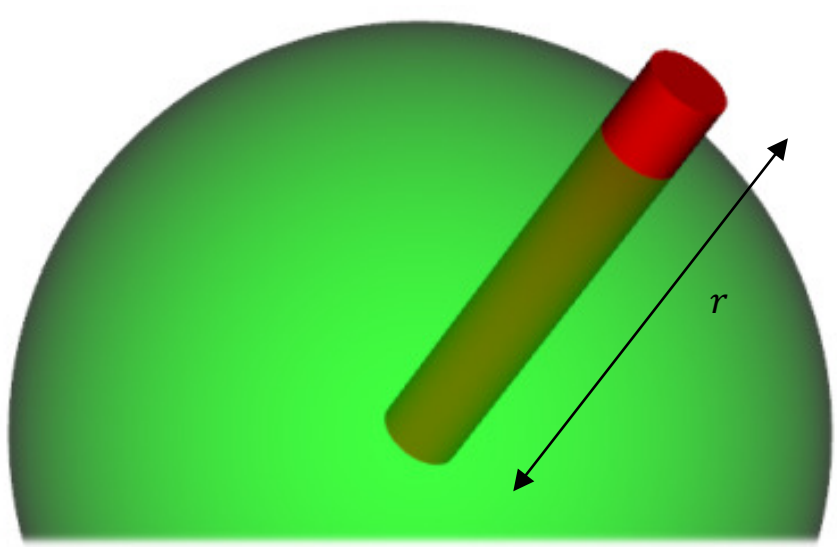

(b)

Fig. 1. Radial integration of the PDF, (a) in a cone of constant solid angle (i.e., the factor $r^{2}$ is considered), and (b) by linear projection (i.e., inaccurately without the factor $r^{2}$ as commonly done in the HARDI literature before this work). 


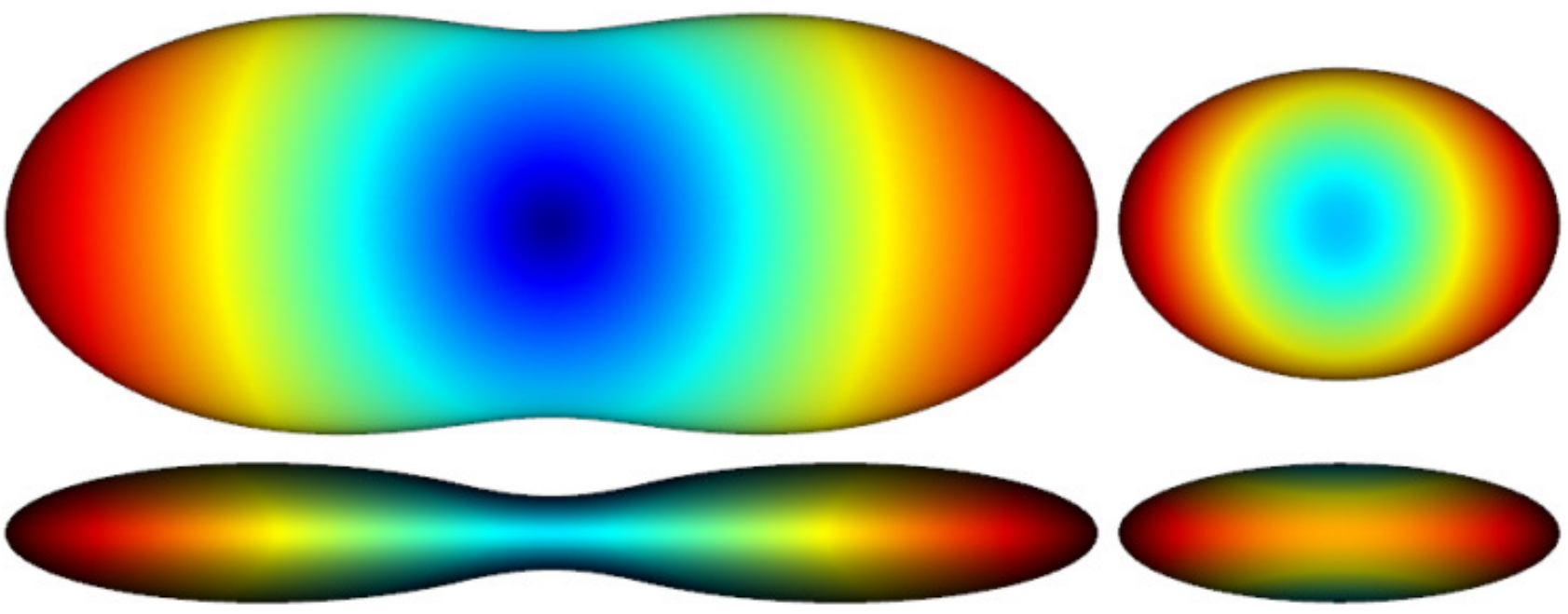

Fig. 2. DTI example of ODF reconstruction (with $\{10,5,1\}$ as the diagonal entries of the tensor), shown from two view angles, (left) with the factor $\boldsymbol{r}^{2}$, (right) without the factor $\boldsymbol{r}^{2}$ and after normalization. Note how less sharp the latter is and how incompletely it represents the true structure of the $O D F$. 

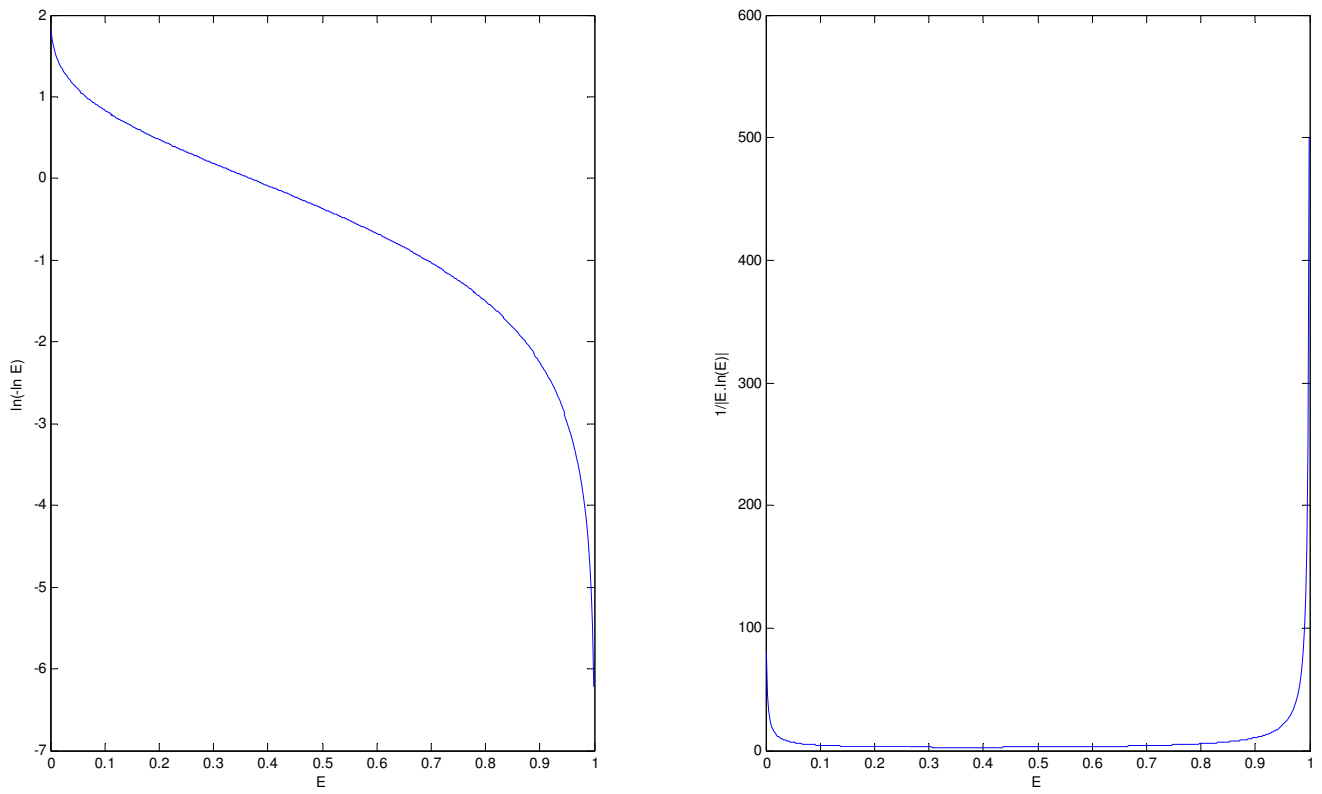

Fig. 3. Behavior of $\ln (-\ln E)$ (left) and the absolute value of its derivative (right) with respect to E. Note how unstable they are for $E$ close to 0 or 1 . 


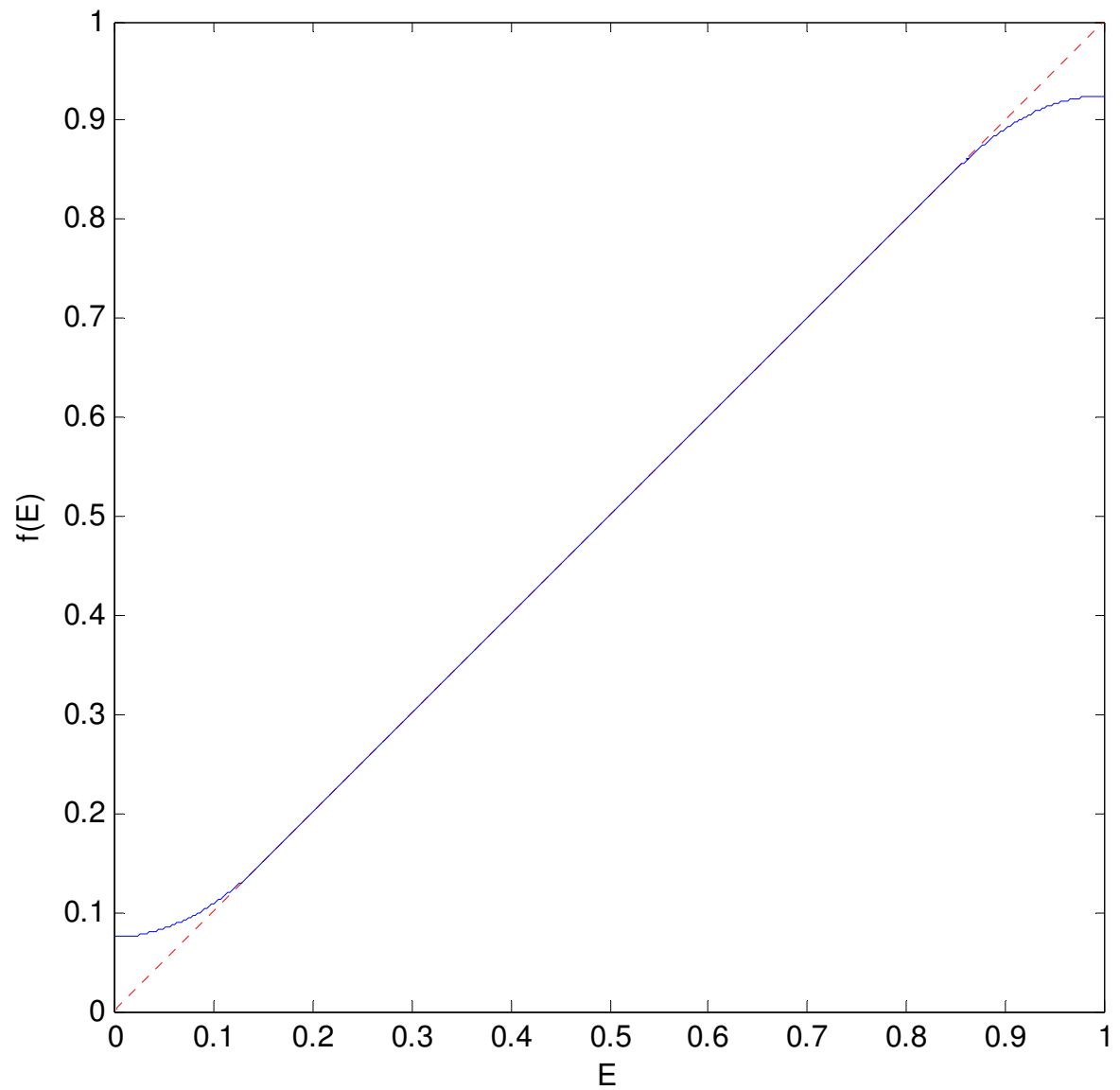

Fig. 4. The regularization function used for the diffusion signal to avoid the unstable regions (blue curve). 


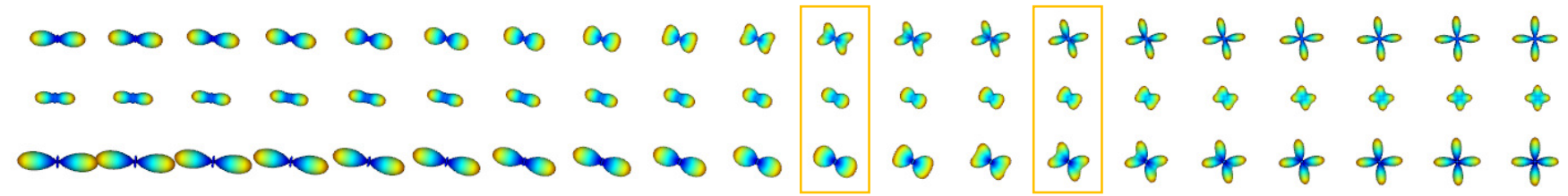

Fig. 5. Experimental results on synthetic data with fiber crossing, using: (top) our proposed technique, (middle) original QBI after normalization, and (bottom) original QBI with Laplace-Beltrami sharpening. 

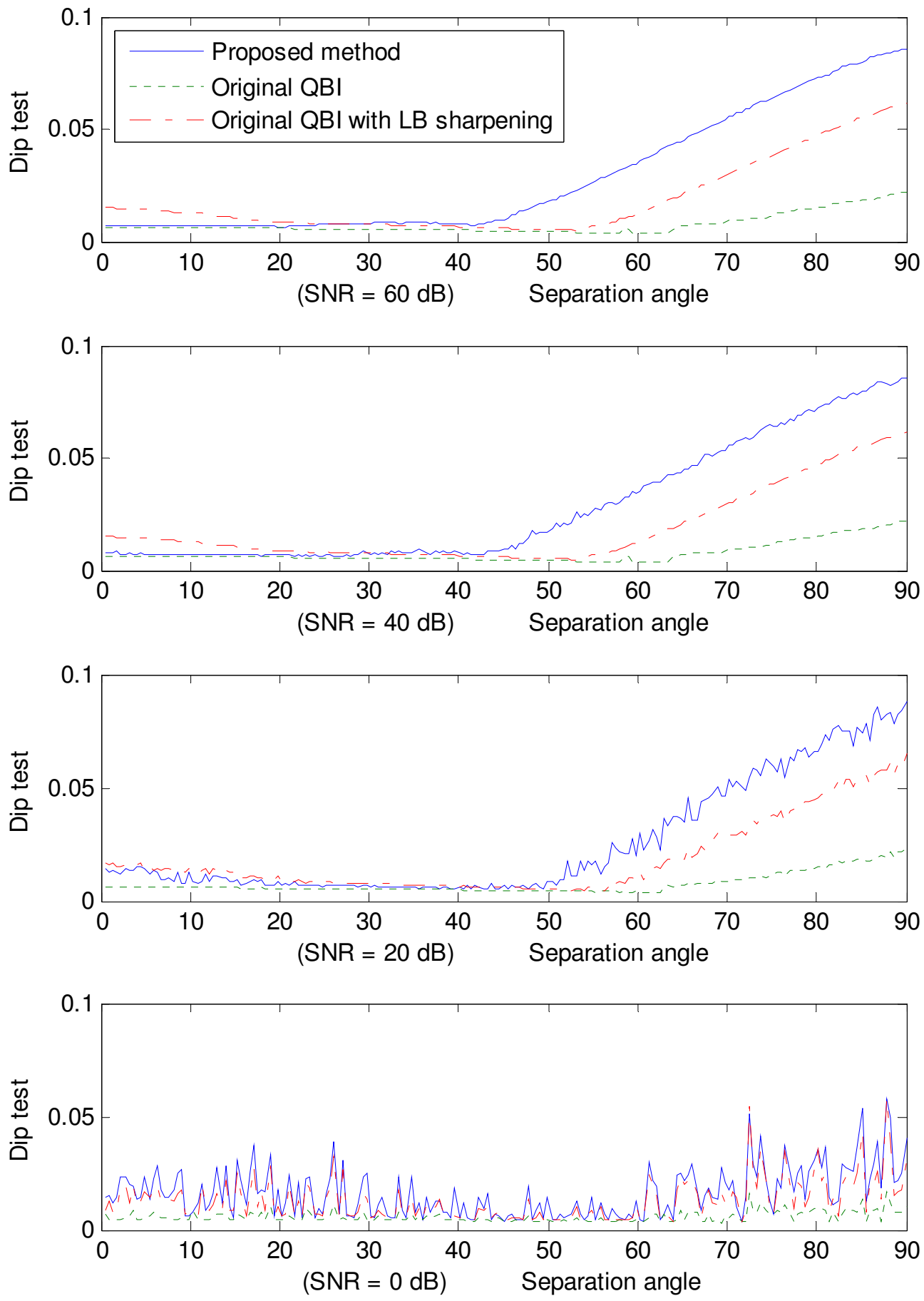

Fig. 6. Results of the dip test on the same ODFs as in Fig. 5, with various noise levels. 


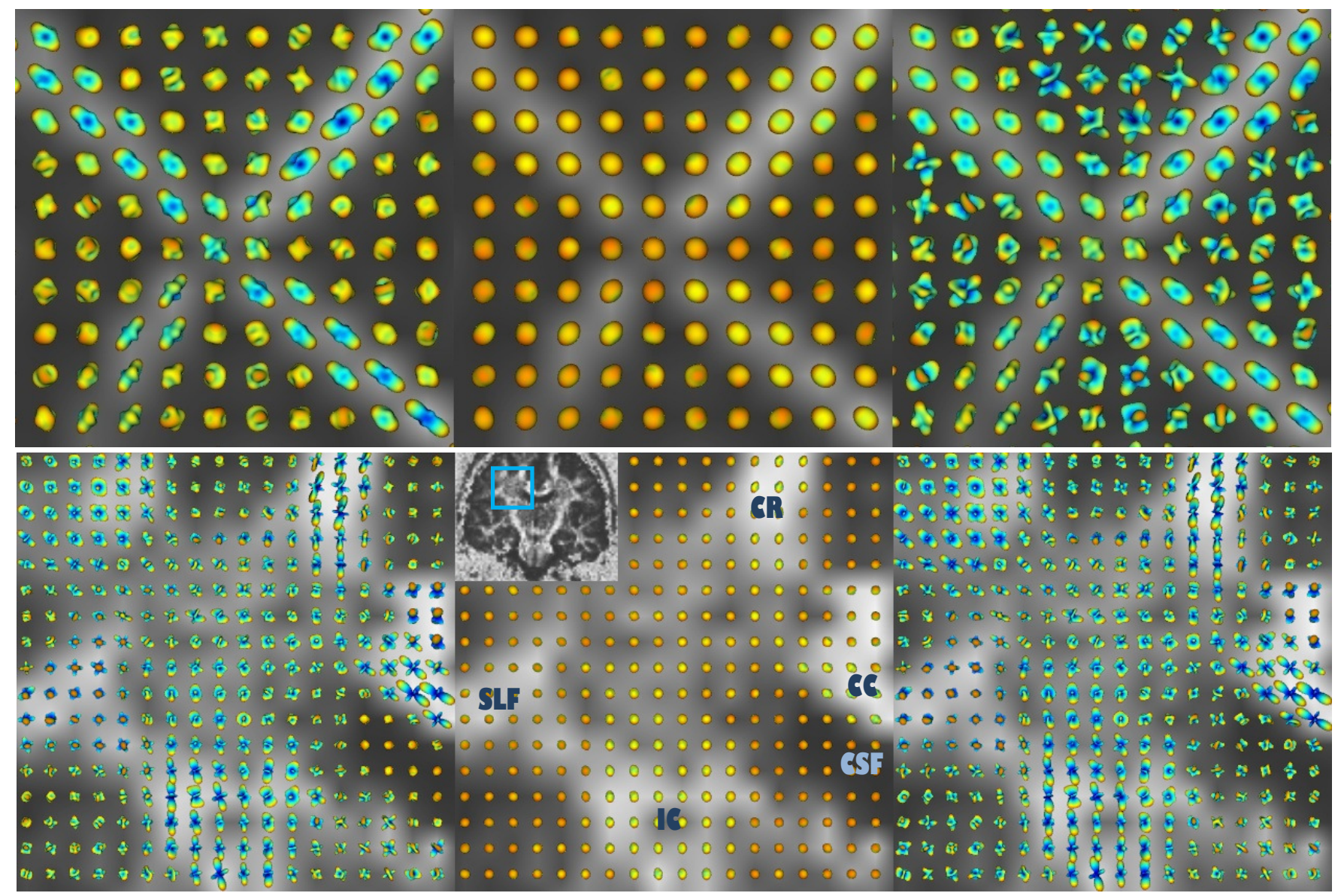

Fig. 7. Reconstructed ODFs from rat spinal cord phantom (top) and human brain (bottom), shown on the GFA map, using: (left) our proposed technique, (middle) original QBI after normalization, and (right) original QBI with Laplace-Beltrami sharpening. 


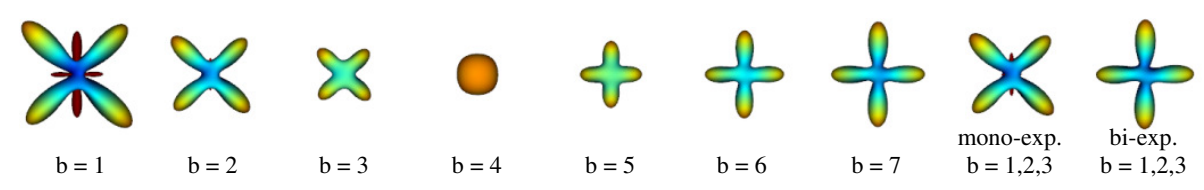

Fig. 8. Results of the ODF reconstruction on synthetic data. Note how the bi-exponential model correctly resolves the maxima of the ODF from low b-values. 


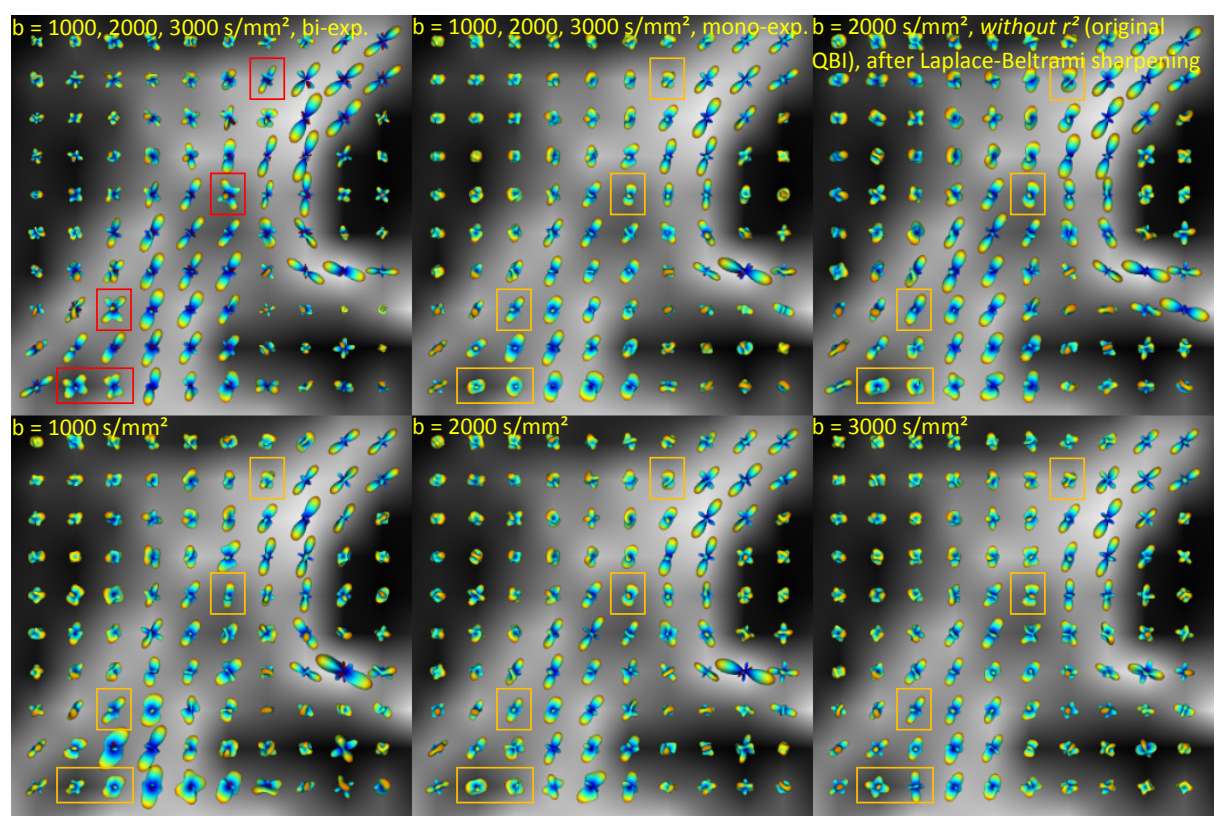

Fig. 9. Reconstructed ODFs from the real brain data, shown on the FA map. The bi-exponential model ODFs (top, left) have been scaled down 1.5 times for better comparison. All the ODFs except those in (top, right) have been reconstructed considering the factor $r^{2}$. Note how the biexponential model for diffusion improves the resolution of fiber crossings, compared to the mono-exponential (constant ADC) model. 